\title{
An interesting matrix equation
}

Aiping Wang, Jiong Sun, and Anton Zettl 


\title{
AN INTERESTING MATRIX EQUATION
}

\author{
AIPING WANG, JIONG SUN, AND ANTON ZETTL
}

Received 17 June, 2008

\begin{abstract}
We study the solutions of a special matrix equation, particularly, their eigenvalues. These matrix solutions have an interesting relationship to unitary matrices.

2000 Mathematics Subject Classification: 47E05, 15A24, 15A18

Keywords: matrix equation, eigenvalues, self-adjoint domains, differential operators, characterization of domains
\end{abstract}

\section{INTRODUCTION}

In this note, we study the matrix equation

$$
A E A^{*}=E,
$$

where

$$
E=\left((-1)^{r} \delta_{r, n+1-j}\right)_{r, j=1}^{n}, \quad n=2 k, 1 \leq k<+\infty,
$$

$\delta_{r, j}$ is the Kronecker delta, and $A$ is an $n \times n$ matrix over the complex numbers $\mathbb{C}$. This study is motivated by the theory of differential operators where this equation plays a critical role in the characterization of self-adjoint domains [1-4].

\section{Eigenvalues}

In this section we characterize the eigenvalues of matrix solutions of equation (1.1). Let $\mathbb{1}$ denote the identity matrix.

Lemma 1. We have

$$
E E=-\mathbb{1}, \quad E^{*}=E^{-1}=-E, \quad \operatorname{det}(E)=1 .
$$

If $A$ is a solution of (1.1), then

$$
\operatorname{det}(A)= \pm 1
$$

The research of the first and second authors was supported by the National Nature Science Foundation of China (Grant No. 10561005), the Doctor Discipline Fund of the Ministry of Education of China (Grant No. 20040126008), and the import talent startup research fund of Tianjin University of Science and Technology (Grant No. 20060433). 
Proof. The properties of $E$ follow from a direct computation. For (2.2) note that $\operatorname{det}(A)=\operatorname{det}\left(A^{*}\right)$ and thus

$$
\operatorname{det}\left(A E A^{*}\right)=\operatorname{det}(A) \operatorname{det}(E) \operatorname{det}\left(A^{*}\right)=[\operatorname{det}(A)]^{2}=1 .
$$

Remark 1 . The simple examples $A=\mathbb{1}$ and $A=i \mathbb{1}$ show that both signs can occur in (2.2).

Lemma 2. If $A$ is a solution of (1.1), then*

$$
\sigma\left(A^{-1}\right)=\sigma\left(A^{*}\right) .
$$

Proof. From (1.1) and (2.1) we get $A E A^{*} E=E E=-\mathbb{1}$. Hence $(A E)^{-1}=$ $-A^{*} E$ and $A^{*}=-E A^{-1} E, A^{-1}=-E A^{*} E$. Therefore,

$$
\begin{aligned}
\operatorname{det}\left(A^{-1}-\lambda \mathbb{1}\right) & =\operatorname{det}\left(-E A^{*} E-\lambda E(-E) \mathbb{1}\right) \\
& =\operatorname{det}(-E) \operatorname{det}\left(A^{*}-\lambda \mathbb{1}\right) \operatorname{det}(E) \\
& =\operatorname{det}\left(A^{*}-\lambda \mathbb{1}\right),
\end{aligned}
$$

and (2.4) follows.

Remark 2. Of course (2.4) holds for any unitary matrix $U$, because $U^{*}=U^{-1}$. All eigenvalues of a unitary matrix lie on the unit circle of the complex plane. Below we derive the corresponding result for matrix solutions $A$ of (1.1). We will see that the eigenvalues of $A$ need not lie on the unit circle but those which do are related to each other in interesting ways and also those which are not on the unit circle have interesting relationships to each other.

Let the spectrum of $A$ have the form

$$
\sigma(A)=\left\{\lambda_{j}=r_{j} e^{i \theta_{j}}: j=1, \ldots, n\right\},
$$

where $r_{j}>0$ and $-\pi<\theta_{j} \leq \pi$ for $j=1, \ldots, n$. We can now give our main result.

Theorem 1. Let $A$ be a solution of (1.1). Then the following properties hold:

(1) If, for some $j$, an eigenvalue $\lambda_{j}$ of $A$ is not on the unit circle, i.e., $r_{j} \neq 1$, then $\frac{1}{r_{j}} e^{i \theta_{j}}$ is also an eigenvalue of $A$.

(2) Let $l$ be the number of pairs of eigenvalues of $A$ not on the unit circle, given by (1) and counting multiplicity, then $l$ can be any one of the numbers $0,1,2, \ldots, k$.

(3) Assume that there exist $l(0 \leq l \leq k)$ pairs of eigenvalues

$$
r_{1} e^{i \theta_{1}}, \frac{1}{r_{1}} e^{i \theta_{1}}, \ldots, r_{l} e^{i \theta_{l}}, \frac{1}{r_{l}} e^{i \theta_{l}}
$$

*As usual, the symbol $\sigma(A)$ stands for the spectrum of $A$. 
of $A$ which are not on the unit circle, where $r_{j}>0, r_{j} \neq 1, j=0,1, \ldots, l$ $(j=0$ means that there are no such eigenvalues, i.e., all eigenvalues are on the unit circle), then there exist $d=2(k-l)$ eigenvalues of $A$,

$$
e^{i \theta_{l+1}}, \cdots, e^{i \theta_{l+d}}
$$

on the unit circle and $\theta_{j}$ satisfy the relations

$$
\sum_{j=1}^{l} 2 \theta_{j}+\sum_{j=1}^{d} \theta_{l+j} \in\{j \pi: j=0, \pm 2, \ldots, \pm 2(k-1), 2 k\}
$$

if $\operatorname{det}(A)=1$, and the relations

$$
\begin{aligned}
& \qquad \sum_{j=1}^{l} 2 \theta_{j}+\sum_{j=1}^{d} \theta_{l+j} \in\{j \pi: j= \pm 1, \pm 3, \pm 5, \ldots, \pm(n-1)\} \\
& \text { if } \operatorname{det}(A)=-1 .
\end{aligned}
$$

Proof. By Lemma 2, we have $\sigma\left(A^{-1}\right)=\sigma\left(A^{*}\right)$, i. e.,

$$
\left\{\frac{1}{\lambda_{j}}: j=1, \ldots, n\right\}=\left\{\bar{\lambda}_{h}: h=1, \ldots, n\right\} .
$$

Therefore, for any $\bar{\lambda}_{h} \in \sigma\left(A^{*}\right)$, there exist $\frac{1}{\lambda_{j}}$ such that $\bar{\lambda}_{h}=\frac{1}{\lambda_{j}}$, i. e.,

$$
r_{h} e^{-i \theta_{h}} r_{j} e^{i \theta_{j}}=1 \text {. }
$$

Hence, $r_{h} r_{j}=1$ and $\theta_{j}=\theta_{h}$ (or $\theta_{j}-\theta_{h}=2 \pi$ ). Therefore, if for some $j, r_{j} e^{i \theta_{j}}$ with $r_{j}>0,-\pi<\theta_{j} \leq \pi$, and $r_{j} \neq 1$ is an eigenvalue of $A$, then $\frac{1}{r_{j}} e^{i \theta_{j}}$ is also an eigenvalue of $A$.

By (1), the eigenvalues not on the unit circle occur in pairs where each pair has the same angle and the conclusion follows.

Conditions (2.6) and (2.7) follow from the fact that $\operatorname{det}(A)=\prod_{j=1}^{n} \lambda_{j}= \pm 1$.

Remark 3. Let $A$ satisfy (1.1). Then $A$ may have all its eigenvalues on the unit circle or there may be none on the unit circle. In all other cases there are an even number of eigenvalues not on the unit circle and these can be paired so that any one pair has the same angle; the angles of the eigenvalues are related to each other according to (2.6) and (2.7). The examples in the next section will illustrate these points further.

\section{EXAMPLES}

In this section we give some examples to illustrate Theorem 1.

Example 1. Let $n=2$. In the second order case either both eigenvalues are on the unit circle or neither one is.

CASE 1. Both eigenvalues are on the unit circle, i. e., $\sigma(A)=\left\{e^{i \theta_{1}}, e^{i \theta_{2}}\right\}$. 
(1) When $\operatorname{det}(A)=1$, we have $e^{i\left(\theta_{1}+\theta_{2}\right)}=1$ and $\theta_{2}=-\theta_{1}$ or $\theta_{1}+\theta_{2}=2 \pi$. Therefore, $\sigma(A)=\left\{e^{i \theta}, e^{-i \theta}\right\}$. Note that the eigenvalues of $A$ are symmetric with respect to the real axis. When $\lambda=1$ or $\lambda=-1$ is an eigenvalue, then it must have multiplicity 2 .

(2) If $\operatorname{det}(A)=-1$, then we have $\theta_{1}+\theta_{2}=\pi$ and the eigenvalues of $A$ are symmetric about the imaginary axis. When $\lambda=i$ or $\lambda=-i$ is an eigenvalue, then it must be a double eigenvalue.

CASE 2. The eigenvalues of $A$ are not on the unit circle, i. e., $\sigma(A)=\left\{r e^{i \theta}, \frac{1}{r} e^{i \theta}\right\}$, where $r>0$ and $r \neq 1$.

(1) When $\operatorname{det}(A)=1$, we have $e^{i(2 \theta)}=1$ and $\theta=0$ or $\pi$. Then the eigenvalues of $A$ are $r$ and $\frac{1}{r}$, or $-r$ and $-\frac{1}{r}$.

(2) If $\operatorname{det}(A)=-1$, then we have $e^{i(2 \theta)}=-1$ and $\theta=\frac{\pi}{2}$ or $-\frac{\pi}{2}$. Then the eigenvalues of $A$ are $r i$ and $\frac{1}{r} i$, or $-r i$ and $-\frac{1}{r} i$.

Example 2. Assume that $n=4$. Then exactly one of the following three cases must hold:

(1) All the eigenvalues are on the unit circle, i. e., $\sigma(A)=\left\{e^{i \theta_{1}}, e^{i \theta_{2}}, e^{i \theta_{3}}, e^{i \theta_{4}}\right\}$, where the numbers $\theta_{i}$ are such that $\sum_{j=1}^{4} \theta_{j}=0, \pm 2 \pi, 4 \pi$ if $\operatorname{det}(A)=1$, and $\sum_{j=1}^{4} \theta_{j}= \pm \pi, \pm 3 \pi$ if $\operatorname{det}(A)=-1$.

(2) One pair of eigenvalues is not on the unit circle and the other two eigenvalues are on the unit circle, i.e.,

$$
\sigma(A)=\left\{e^{i \theta_{1}}, e^{i \theta_{2}}, r e^{i \theta}, \frac{1}{r} e^{i \theta}\right\}
$$

where $r>0$ and $r \neq 1$. In this case $\theta_{1}, \theta_{2}$, and $\theta$ satisfy $\theta_{1}+\theta_{2}+2 \theta=$ $0, \pm 2 \pi, 4 \pi$ when $\operatorname{det}(A)=1$, and $\theta_{1}+\theta_{2}+2 \theta= \pm \pi, \pm 3 \pi$ when $\operatorname{det}(A)=$ -1 .

(3) No eigenvalue of $A$ is on the unit circle. In this case there are two pairs of eigenvalues each pair having the same angle, i. e.,

$$
\sigma(A)=\left\{r_{1} e^{i \theta_{1}}, \frac{1}{r_{1}} e^{i \theta_{1}}, r_{2} e^{i \theta_{2}}, \frac{1}{r_{2}} e^{i \theta_{2}}\right\},
$$

where $r_{j}>0$ and $r_{j} \neq 1$, and the numbers $\theta_{1}, \theta_{2}$ satisfy $\theta_{1}+\theta_{2}=0, \pm \pi, 2 \pi$ when $\operatorname{det}(A)=1$, and $\theta_{1}+\theta_{2}= \pm \frac{\pi}{2}, \pm \frac{3 \pi}{2}$ when $\operatorname{det}(A)=-1$. Here $\theta_{1}$ and $\theta_{2}$ are not necessarily distinct.

Example 3. Assume that $n=6$. There are exactly four subcases:

(1) All eigenvalues are on the unit circle, i. e.,

$$
\sigma(A)=\left\{e^{i \theta_{1}}, e^{i \theta_{2}}, e^{i \theta_{3}}, e^{i \theta_{4}}, e^{i \theta_{5}} e^{i \theta_{6}}\right\},
$$


where the numbers $\theta_{j}$ satisfy $\sum_{j=1}^{6} \theta_{j}=0, \pm 2 \pi, \pm 4 \pi, 6 \pi$ if $\operatorname{det}(A)=1$, and $\sum_{j=1}^{6} \theta_{j}= \pm \pi, \pm 3 \pi, \pm 5 \pi$, if $\operatorname{det}(A)=-1$.

(2) One pair is not on the unit circle and the other four eigenvalues are on the unit circle , i. e.,

$$
\sigma(A)=\left\{e^{i \theta_{1}}, e^{i \theta_{2}}, e^{i \theta_{3}}, e^{i \theta_{4}}, r e^{i \theta}, \frac{1}{r} e^{i \theta}\right\},
$$

where $r>0, r \neq 1$, and the numbers $\theta_{j}$ and $\theta$ satisfy

$$
\sum_{j=1}^{4} \theta_{j}+2 \theta=0, \pm 2 \pi, \pm 4 \pi, 6 \pi
$$

when $\operatorname{det}(A)=1$, and

$$
\sum_{j=1}^{4} \theta_{j}+2 \theta= \pm \pi, \pm 3 \pi, \pm 5 \pi
$$

when $\operatorname{det}(A)=-1$.

(3) Two pairs are not on the unit circle and the other two eigenvalues are on the unit circle, i. e.,

$$
\sigma(A)=\left\{e^{i \theta_{1}}, e^{i \theta_{2}}, r_{1} e^{i \theta_{3}}, \frac{1}{r_{1}} e^{i \theta_{3}}, r_{2} e^{i \theta_{4}}, \frac{1}{r_{2}} e^{i \theta_{4}}\right\},
$$

where $r_{j}>0$ and $r_{j} \neq 1$, and the numbers $\theta_{j}$ satisfy

$$
\theta_{1}+\theta_{2}+2 \theta_{3}+2 \theta_{4}=0, \pm 2 \pi, \pm 4 \pi, 6 \pi
$$

when $\operatorname{det}(A)=1$, and

$$
\theta_{1}+\theta_{2}+2 \theta_{3}+2 \theta_{4}= \pm \pi, \pm 3 \pi, \pm 5 \pi \text {. }
$$

when $\operatorname{det}(A)=-1$.

(4) No eigenvalue is on the unit circle, i. e.,

$$
\sigma(A)=\left\{r_{1} e^{i \theta_{1}}, \frac{1}{r_{1}} e^{i \theta_{1}}, r_{2} e^{i \theta_{2}}, \frac{1}{r_{2}} e^{i \theta_{2}}, r_{3} e^{i \theta_{3}}, \frac{1}{r_{3}} e^{i \theta_{3}}\right\},
$$

where $r_{j}>0$ and $r_{j} \neq 1$, and the numbers $\theta_{j}$ satisfy

$$
\sum_{j=1}^{3} \theta_{j}=0, \pm \pi, \pm 2 \pi, 3 \pi
$$

when $\operatorname{det}(A)=1$, and

$$
\sum_{j=1}^{3} \theta_{j}= \pm \frac{\pi}{2}, \pm \frac{3 \pi}{2}, \pm \frac{5 \pi}{2}
$$

when $\operatorname{det}(A)=-1$. 
Example 4. Let

$$
A=\left(\begin{array}{cccccc}
r_{1} e^{i \theta_{1}} & 0 & 0 & 0 & 0 & 0 \\
0 & r_{2} e^{i \theta_{2}} & 0 & 0 & 0 & 0 \\
0 & 0 & r_{3} e^{i \theta_{3}} & 0 & 0 & 0 \\
0 & 0 & 0 & \frac{1}{r_{3}} e^{i \theta_{3}} & 0 & 0 \\
0 & 0 & 0 & 0 & \frac{1}{r_{2}} e^{i \theta_{2}} & 0 \\
0 & 0 & 0 & 0 & 0 & \frac{1}{r_{1}} e^{i \theta_{1}}
\end{array}\right)
$$

Then $A$ satisfies (1.1) for any $r_{j}>0$ and $\theta_{j} \in(-\pi, \pi]$. Choosing $r_{j}=1$ for $j=1,2,3$ all six eigenvalues are on the unit circle and their angles satisfy the equations (3.1) or (3.2). By choosing three distinct $\theta_{j}$ we get three distinct eigenvalues each with multiplicity 2 , by choosing $\theta_{1}=\theta_{2} \neq \theta_{3}$ we get two distinct eigenvalues, one having multiplicity 2 , the other 4 . Finally by choosing $\theta_{1}=\theta_{2}=\theta_{3}$ we get one eigenvalue with multiplicity 6 . Clearly this example extends to $n=2 k$ for $k>3$ and can be used to show that the eigenvalues on the unit circle can have multiplicity $m$ for any $m, m=2,4,6, \ldots, 2 k$.

Remark 4. Just as in the above examples, for $n=2 k$ there are exactly $k+1$ cases ranging from exactly $j$ pairs not on the unit circle for $j=1,2, \ldots, k$ to all eigenvalues on the unit circle.

Remark 5. It is interesting to compare matrices satisfying (1.1) with unitary matrices. Clearly equation (1.1) is equivalent with

$$
(A E)(E A)^{*}=\mathbb{1}
$$

which can be compared to

$$
U U^{*}=\mathbb{1},
$$

but as Theorem 1 shows there are major differences, as well as similarities, in the behavior of the eigenvalues of the matrices satisfying these two equations. Lemma 1 holds for unitary matrices since $U^{-1}=U^{*}$ but all the eigenvalues of a unitary matrix lie on the unit circle. The fact that one $E$ in (3.4) is "on the wrong side" is significant.

\section{ACKNOWLEDGEMENTS}

The first two authors are grateful to the Mathematics Department of Northern Illinois University, where some part of this work was carried out, for its hospitality.

\section{REFERENCES}

[1] W. N. Everitt and L. Markus, Boundary value problems and symplectic algebra for ordinary differential and quasi-differential operators, ser. Mathematical Surveys and Monographs. Providence, RI: American Mathematical Society, 1999, vol. 61. 
[2] M. A. Naimark, Linear differential operators. Part II: Linear differential operators in Hilbert space, ser. With additional material by the author, and a supplement by V. Ė. Ljance. Translated from the Russian by E. R. Dawson. English translation edited by W. N. Everitt. New York: Frederick Ungar Publishing Co., 1968.

[3] A. Wang, J. Sun, and A. Zettl, "Characterization of domains of self-adjoint ordinary differential operators," preprint.

[4] A. Zettl, Sturm-Liouville theory, ser. Mathematical Surveys and Monographs. Providence, RI: American Mathematical Society, 2005, vol. 121.

Authors' addresses

\section{Aiping Wang}

Mathematical Department, Tianjin University of Science and Technology, Tianjin 300457, China

E-mail address: aipingetust.edu.cn

\section{Jiong Sun}

Mathematical Department, Inner Mongolia University, Hohhot 010021, China

E-mail address: masun@imu.edu.cn

\section{Anton Zettl}

Mathematical Department, Northern Illinois University, DeKalb, Il. 60115, USA

E-mail address: zettl@math.niu.edu 